\title{
Improved understanding of gastrointestinal stromal tumors biology as a step for developing new diagnostic and therapeutic schemes (Review)
}

\author{
MARTA MAGDALENAFUDALEJ ${ }^{1,2}$ and ANNA MARIA BADOWSKA-KOZAKIEWICZ ${ }^{1}$ \\ ${ }^{1}$ Department of Cancer Prevention, ${ }^{2}$ Doctoral School, Medical University of Warsaw, 02-091 Warsaw, Poland
}

Received December 23, 2020; Accepted February 10, 2021

DOI: $10.3892 / \mathrm{ol} .2021 .12678$

\begin{abstract}
A gastrointestinal stromal tumor (GIST) is the most common mesenchymal tumor of the human gastrointestinal tract, with an estimated incidence of $10-15$ per 1 million per year. While preparing holistic care for patients with GIST diagnosis, scientists might face several difficulties - insufficient risk stratification, acquired or secondary resistance to imatinib, or the need for an exceptional therapy method associated with wild-type tumors. This review summarizes recent advances associated with GIST biology that might enhance diagnostic and therapeutic strategies. New molecules might be incorporated into risk stratification schemes due to their proven association with outcomes; however, further research is required. Therapies based on the significant role of angiogenesis, immunology, and neural origin in the GIST biology could become a valuable enhancement of currently implemented treatment schemes. Generating miRNA networks that would predict miRNA regulatory functions is a promising approach that might help in better selection of potential biomarkers and therapeutical targets in cancer, including GISTs.
\end{abstract}

\section{Contents}

1. Introduction

2. Angiogenic markers

3. Origin from Cajal cells

4. MicroRNAs

5. Immune system

6. Raf kinase inhibitory protein (RKIP)

7. Epithelial-to-mesenchymal transition (EMT)

8. Conclusion

Correspondence to: Dr Marta Magdalena Fudalej, Department of Cancer Prevention, Medical University of Warsaw, 81 Żwirki i Wigury Street, 02-091 Warsaw, Poland

E-mail:mmfudalej@gmail.com

Key words: gastrointestinal stromal tumor, oncology, angiogenesis, microRNA, carcinogenesis

\section{Introduction}

A gastrointestinal stromal tumor (GIST) is the most common mesenchymal tumor of the human gastrointestinal tract, with an estimated incidence of 10-15 per 1 million per year (1-5). Approximately $90 \%$ of GISTs are located in the stomach and small intestine, with gastric lesions being the most prevalent $(\sim 60 \%)(1,3,6,7)$. GIST occurs with similar frequency in males and females $(6,8)$; nevertheless, some studies claim slight predominance in males (1). Patients might be diagnosed with GISTs at any age, yet they rarely occur $(0.5 \%)$ in individuals younger than 20 years. The median age of detection is estimated to be 65 years of age $(1,2,6,8)$.

Generally, patients presenting with GIST are asymptomatic; nevertheless, some demonstrate non-specific symptoms such as abdominal distension, pain, nausea or vomiting $(1,8)$. The median tumor size at diagnosis is $\sim 6 \mathrm{~cm}$; however, it may reach $20 \mathrm{~cm}$ (8). Although nodal metastases rarely follow a primary tumor, distant metastasis encompassing the abdominal cavity or the liver concern $\sim 20 \%$ of patients at diagnosis $(8,9)$. The leading treatment for GIST cases remains surgical resection (10). Standard first-line therapy for inoperable, metastatic or recurrent issues is the tyrosine-kinase inhibitor imatinib (11).

Some of the most critical GIST carcinogenesis mutations occur in the tyrosine kinase family (KIT) or platelet-derived growth factor receptor A (PDGFRA) gene. Only a small proportion of GISTs seems to be associated with neither KIT nor PDGFR sporadic mutations and is assigned to the wild-type (WT) group (5). KIT and PDGFRA mutation types might predict advanced or metastatic GIST response to imatinib (12). GISTs with KIT exon 11 mutations are the most sensitive to imatinib treatment, whereas KIT exon 9 mutations require a higher dosage of this inhibitor. On the other hand, cases with PDGRA D842V mutation are imatinib-resistant $(7,12)$. Despite the significant improvement in disease control and overall survival (OS) in advanced GIST cases associated with imatinib usage, patients frequently suffer from acquired or secondary resistance. Therefore, it appears essential to identify the mechanism underlying the resistance to develop an intervention that might be applied in this group of patients $(13,14)$.

Risk stratification of GISTs attempts to evaluate the risk of an unfavorable outcome and select patients who may benefit from adjuvant therapy (15). Various risk stratification 
systems have evolved over the years, but none is proved superior to the other (6). The first risk stratification was proposed by Schaefer et al (12), predicting GIST malignant behavior by classification into very low, low, intermediate and high-risk categories based on tumor size and mitotic rate. One of the widely used classification, Armed Force Institute of Pathology (AFIP) risk classification, is based on primary tumor site (extra-gastric location has worse predicted outcome), mitotic count and primary tumor size (16). Notwithstanding this, all risk assessments present one common drawback concerning the non-linear continuous character of variables such as tumor size and mitotic count (17). Moreover, behaviors of specific GIST subgroups [for example, succinate dehydrogenase (SDH) deficiency] are less well predicted by all systems (18).

All things considered, while preparing holistic care for patients with GIST diagnosis, scientists might face several difficulties: Insufficient risk stratification, acquired or secondary resistance to imatinib or the need for an exceptional therapy method associated with wild-type tumors. The present review summarizes recent advances associated with GIST biology that might enhance diagnostic and therapeutic strategies. The described area embraces angiogenesis, immunology, epithelial-to-mesenchymal transition, origin from Cajal cells, microRNAs, and Raf kinase inhibitory proteins. According to the authors' best knowledge, similar reviews encompassing the described area have not been published yet.

\section{Angiogenic markers}

Angiogenesis is proved to be one of the principal processes in tumor growth and metastasis promotion. It is regulated by a balance of angiogenic and anti-angiogenic cytokines (19). Anti-angiogenic strategies are an area of great interest throughout the scientific world. They might be categorized into three groups: i) Protein-based immunotherapeutics directly neutralizing vascular endothelial growth factor (VEGF) (Bevacizumab); ii) receptor tyrosine kinase inhibitors (Sunitinib); and iii) antagonists of the mammalian target of rapamycin (Everolimus) (20).

Anti-angiogenic therapies eradicate the existing tumor vessels and obstruct the formation of new ones and consequently avert the tumor cell's nutrition. Moreover, such strategies decrease the degree of malignancy and increase the efficiency of conventional treatment. Notwithstanding this, single inhibition of VEGF receptors or tyrosine kinase receptors is insufficient for hindering the entire angiogenesis process and might develop an adaptive resistance towards treatment, partly due to changes in the immune microenvironment of the tumor (21). Despite their defects, anti-angiogenic therapies present high potential and need further research, encompassing specific molecules that may contribute to establishing highly effective personalized oncological treatment.

As far as GIST is concerned, the enormous role of angiogenesis in tumor progression is verified by the high efficiency of second-line Sunitinib. The primary mechanism of Sunitinib targets multiple receptor tyrosine kinases, including these for VEGF-critical mediators of angiogenesis (13).

VEGF expression is frequently increased in GISTaccounted positively for $60-80 \%$ of all studied cases (22-24). Null or weak expression of VEGF is associated with better prognosis [higher progression-free survival (PFS) and OS], independently of the tumor genotype. Moreover, low VEGF expression is associated with a high therapeutic response to imatinib mesylate $(13,24)$. In general, GIST cells do not express VEGF-C, playing a critical role in node metastasis via lymphangiogenesis (25). The lack of VEGF-C expression could be one of the pivotal mechanisms explaining the rare occurrence of lymph node metastases among patients with GIST diagnosis (26).

Among other angiogenic factors, endoglin (CD105) and platelet endothelial cell adhesion molecule (PECAM-1) are considered to be associated with patients outcomes (24). In GISTs, the association between the strong immunohistochemical staining of CD105 with various morphological criteria is associated with a worse prognosis, encompassing a mitotic index above 5 mitoses per 50 high-power fields and a high degree of risk (27). Moreover, the average value for the CD105 and PECAM-1 expression is significantly higher in patients with poor prognosis compared with the group of patients who are presented without recurrence $(24,28)$.

Fibroblast growth factors (FGFs) and their receptors (FGFRs) are known as the potent regulators of angiogenesis (29). Massive secretion of the multiple chemokines, including FGF-2, was discovered to be induced by the imatinib c-KIT inhibition. Increased production of FGF-2 by GIST cells treated with imatinib activates the FGF-2/FGFR autocrine loop that presents a negative impact on the disease progression and might become one of the most important mechanisms underlying imatinib-resistance among some patients with GIST (30). Inhibition of FGF-signaling in imatinib-resistant patients was proved to restore their sensitivity to the applied treatment (31). Moreover, the combined inhibition of KIT and FGFR signaling increases growth inhibition in GIST cells both in vitro and in vivo (32).

Collagen and calcium-binding EGF domain-containing protein 1 (CCBE1) is suggested to function as an independent regulator of budding and migration of lymphangioblasts, and as a result, to promote lymphangiogenesis (33). Notwithstanding this, in the study conducted on GIST tissues, CCBE1 was proved to be specifically located in the vessel wall with co-localization of a marker of vascular endothelial cells CD31 (34). Higher levels of CCBE1 were associated with higher risk groups of GIST, lower survival and were suspected of counteracting the anti-tumor effects of imatinib (34). However, studies conducted on ovarian and breast cancers presented contradictory findings - higher CCBE1 expression was associated with better survival rates (35). In conclusion, CCBE1 action seems to be contextually based upon tumor origin - epithelial or mesenchymal.

\section{Origin from Cajal cells}

Hirota et al (36) found in 1998 that GISTs originate from interstitial cells of Cajal (ICCs) in the myenteric plexus of the alimentary tract. Pacemaker potentials of ICCs suggest that mutations in genes required for synapse and neural development might underlie some GIST behaviors (37). Moreover, the neuroendocrine phenotype of GIST was proved by the presence of synaptic-like micro-vesicle proteins, ghrelin, and peptide hormone receptors in the analyzed GIST specimens (38). 


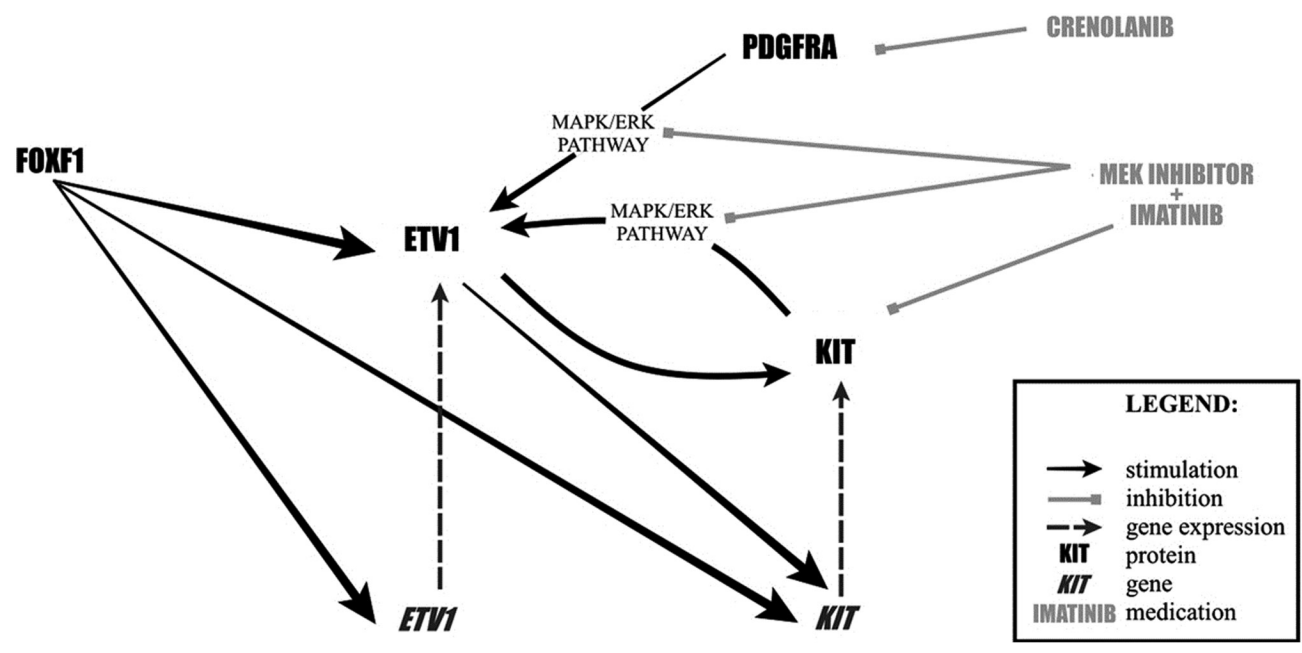

Figure 1. A simplified scheme of dependencies between molecules associated with interstitial cells of Cajal and genes.

ICCs requires the principal signaling regulator-KIT, and a lineage-specific master transcription factor-ETS translocation variant 1 (ETVI), for lineage specification and survival (39). ETV1 is a master regulator of an ICC-GIST-specific transcription network predominantly through enhancer-binding (40). The transcription of KIT and ETVI is directly regulated by the forkhead family member, FOXF1, that co-localizes with ETV1 at enhancers (39). Mutant KIT and ETV1 form a positive feedback loop, in which KIT excessively activates downstream mitogen-activated protein kinase (MAPK) signaling that stabilizes ETV1. In turn, ETV1 consolidates mutant KIT overexpression $(39,40)$. In GIST xenografts, the treatment combination of imatinib and MEK162 (a MEK inhibitor) resulted in a practically complete response with rapid inhibition of the MAPK activity, loss of ETV1 protein, and downregulation of ETV1 target genes. This therapy represents a significantly more effective treatment strategy than imatinib alone and might prevent the development of imatinib-resistance (41). Another therapeutic option includes the inhibition of PDGFRA by crenolanib, a novel potent pharmacological inhibitor of wild-type and oncogenic type of receptor tyrosine kinase III with high selectivity for PDGFRA relative to KIT. The inhibition of PDGFRA disturbs a KIT-EKR-ETV1-KIT signaling loop and promotes proteasomal degradation of ETV1 through decreased ERK-MAPK phosphorylation (42). The protein level not significantly affected by the KIT or MARP pathways perturbations is FOXF1. Notably, FOXF1 loss results in decreased ETV1 protein expression and global loss of ETV1 chromatin binding. It creates a unique therapeutic opportunity to target the cellular context for all GIST cases, including those that do not pose drug-sensitive mutations, such as SDH-deficient ones (Fig. 1) (39).

Contrary to all the aforementioned findings concerning ETV1, in the study by Sakamaki et al (43), ETV1 mRNA expression was negatively associated with malignancy, with detected attenuation in aggressive and malignant cases of GIST. Patients with low ETV1 expression experienced shorter relapse-free survival (RFS) compared with patients with a higher one. Notwithstanding this, the findings aforementioned concerned only ETV1 mRNA, being different from the protein, and a negative feedback system regulating mRNA by the level of ETV1 protein might try to explain the results (43).
Cell adhesion molecules, including Slitrk3 (ST3), are essential for establishing and regulating the synaptic connections (44). The function of ST3 in carcinogenesis remains unclear; however, its expression was detected in GIST tissues in accordance with clinicopathological features (37). ST3 expression was correlated with decreased OS and disease-free survival (DFS) and was proposed as a new enhancement for widely applied AFIP risk stratification classification $(37,45)$.

Cell adhesion molecule L1-like protein (CHL1) is a multidomain type 1 membrane glycoprotein of the immunoglobulin superfamily playing various functions in developing the neuronal system. Its role in cancer cell growth, invasion and migration was demonstrated in numerous studies encompassing different types of malignancies (46). GIST expresses CHL1 on mRNA as well as on protein level. Moreover, systemic CHL1 levels are increased in patients with GIST and is associated with a shortened RFS, regardless of other clinicopathological parameters (47).

Phosphodiesterase 3A (PDE3A) is identified as an ICCs marker playing an influential role in their development; however, not essential for their occurrence (48). PDE3A is found in most GIST samples, regardless of their histological type, and thus, might be suggested as a prospective novel marker for the patient prognosis (49).

\section{MicroRNAs}

MicroRNAs (miRNAs) are small endogenous RNAs that regulate post-transcriptional silencing of target genes (50). Deregulated expression of various miRNAs confers the malignant cells tumorigenic potential. Considering the complexity of miRNAs connections involved in carcinogenesis, focusing on a single miRNA molecule represents a limited clinical approach (51). Emerging evidence highlights that miRNA dysregulation is an essential component in GIST expansion; nevertheless, the entire mechanism remains unclear (52). Some miRNAs (miR-148b-3p, miR-494, miR-218) negatively regulate KIT protein expression and inhibit GIST cell proliferation and invasion (53-55).

On the other hand, miR-218 might improve GIST cells' sensitivity to imatinib through PI3K/AKT signaling 
pathway (56). As far as prognosis is concerned, overexpression of miR-196a and low expression of miR-186 are associated with poorer prognosis in patients with GIST $(57,58)$. As previously mentioned, focusing on a single miRNA brings limited evaluation; however, undoubtedly, miRNAs present an enormous impact on GIST biology. miRNA is suspected of building regulatory networks controlling various cellular functions (59). Generating miRNA networks that would predict miRNAs' regulatory functions is a promising approach that might help select potential biomarkers and therapeutical targets in cancer, including GISTs (51).

Due to the involvement of miRNA in carcinogenesis, therapeutics based on these molecules represent one of the significant areas of scientists' interest. Various candidates have been identified as potential therapeutic applications; nevertheless, there is still much to learn about transforming them into effective, targeted drug delivery systems (60). Commonly, miRNA-based therapeutics are tolerated well in humans in various ongoing cancer-associated clinical trials. The treatment strategy is based mainly on the anti-miRNAs that inhibit the mature miRNAs from binding to their targets and consequently block the participation of these miRNAs in cancer development (61). Leading barriers associated with this therapy are specific delivery platforms to reach the targeted cell or miRNA. Suggested areas that can be used to formulate miRNAs delivery effectively are virus-based carriers (lentiviruses, adenoviruses or adeno-associated viruses), biocompatible and biodegradable liposomes, or nanoparticles (62). Extensive research on the mechanism of pharmacological miRNA targeting and the optimization of application methods might enable the future implementation of miRNA-based therapeutics into the oncological schemes, including those for patients with GIST diagnosis.

\section{Immune system}

Imatinib prolongs patients' survival not only by its direct effect on tumor cells but also by indirect immunostimulatory effects on T and NK cells; thus, appropriate complementary immunotherapy might further improve the patients' outcomes (63). GIST microenvironment presents a suppressed immune system, due to the high infiltration of tumor-associated macrophages (TAMs) that promote tumor development by suppressing Th1-mediated inflammation and stimulating angiogenesis (64).

In a GIST animal model, imatinib increases the activation, proliferation and frequency of intratumoral $\mathrm{CD} 8^{+} \mathrm{T}$ cells and, on the other hand, results in the apoptosis of regulatory $\mathrm{T}$ cells (Tregs) (65). The mechanism underlying this phenomenon might be associated with the overexpression of the enzyme indoleamine 2,3-dioxygenase (IDO) in malignant cells. IDO functions as one of the primary regulators in the biological progression of malignancies by suppressing $\mathrm{T}$ and natural killer (NK) cells, generating and activating Treg cells. Imatinib was proved to decrease IDO expression, leading to $\mathrm{CD}^{+} \mathrm{T}$ cells activation and Tregs apoptosis (66). Moreover, IDO inhibition by imatinib partially accounts for the anti-tumor efficacy of complementary programmed death receptor 1 (PD-1)/programmed cell death 1 ligand 1 (PD-L1) blockade. PD-L1 expression, triggered by interferon- $\gamma(\mathrm{INF}-\gamma)$ has been proven to be an independent factor of poor prognosis in GIST $(67,68)$. Both in vivo and in vitro, anti-PD-1 and anti-PD-L1 had no efficacy when used alone. Still, they enhanced the effectiveness of imatinib by increasing $\mathrm{T}$ cell effector function in the presence of KIT and IDO inhibition $(67,69)$. The primary resistance to PD-1 might be associated with the residence of GIST-associated macrophages expressing IDO1 leading to the immune-suppressive phenotype of malignancy cells (70).

Chemokines are a class of chemotactic cytokines with low molecular mass involved in cancer progression (71). CC chemokine receptor type 8 (CCR8) is one of the most critical chemokine receptors, mainly expressed in Tregs. Its ligand, CCL1, enhances Treg immunosuppressive activity through CCR8 recruitment, with a positive feedback loop (72). In GIST specimens, low expression of CCR8 was positively associated with the patients' survival (10). CCR8 recruits $\mathrm{FOXP} 3^{+}$Treg cells to reveal an immunosuppressive function, which results in a decreased proportion of $\mathrm{CD}^{+}$ $\mathrm{T}$ cells/Tregs and leads to a poor prognosis in solid malignancies $(73,74)$. Interaction between another chemokine receptor, $\mathrm{CXC}$ chemokine receptor (CXCR) 4 and its ligand CXC chemokine ligand (CXCL) 12, is one of the postulated mechanisms leading to the increased organ-specific metastasis development (75). One of the most repeated GIST mutations, KIT exon 11 557-558 deletion, enhances ETV1 and increases CXCR4 expression in GIST cells. Typically, GIST metastases occur in the liver. CXCL12 expressed by hepatic cells attracts GIST cells harboring upregulated expression of CXCR4 (8,71).

\section{Raf kinase inhibitory protein (RKIP)}

Raf kinase inhibitory protein (RKIP) is a highly conserved kinase inhibitor functioning as a metastasis suppressor in various malignancies; thus, its downregulation is proved to be a frequent occurrence in metastatic tumors (76). The high possibility of negative RKIP expression in GISTs is correlated with larger tumor size, despite no association with the number of mitotic figures (77). The lack of RKIP expression restrains the MAPK signaling pathway regulating the cell cycle, resulting in increased proliferation of tumor cells (78). Patients with higher RKIP expression are suspected of having an improved prognosis with higher survival rates; however, it cannot be an independent prognostic factor in GIST $(77,79)$.

\section{Epithelial-to-mesenchymal transition (EMT)}

EMT is a reversible cellular program that transiently changes epithelial cells into a mesenchymal phenotype characterized by loss of apical-basal polarity, reorganization of their cytoskeleton and increased cellular motility. EMT enables cancer cells to fulfill the invasion-metastasis cascade, encompassing local invasion, intravasation and extravasation. On the other hand, to efficiently form macroscopic metastases, carcinoma cells need to revert to a more epithelial phenotype by undergoing mesenchymal-epithelial transition (MET). Pathologists might use the detection of many of the EMT-associated protein markers as highly specific indicators of high-grade malignancy $(80,81)$. 


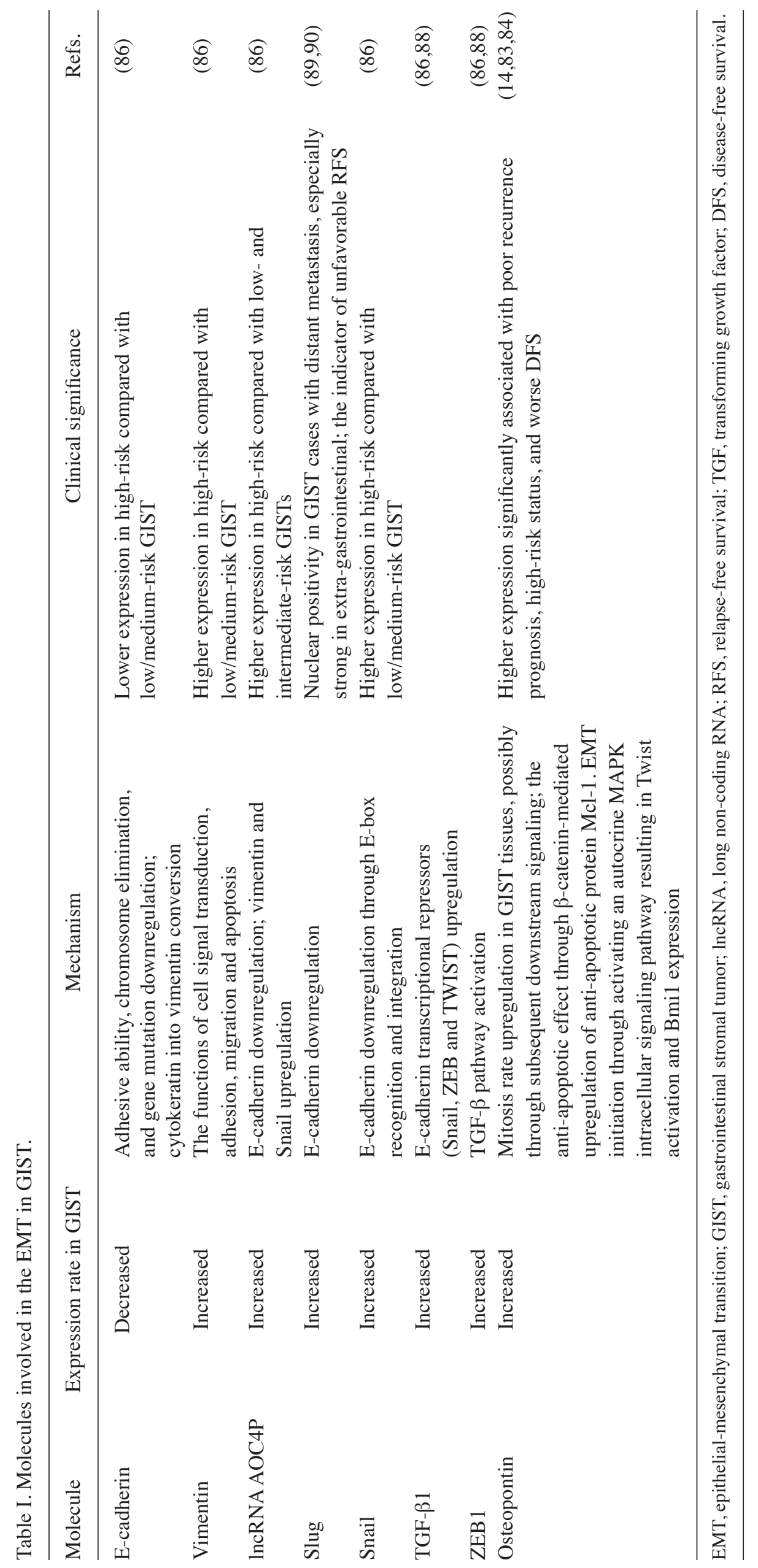


Osteopontin (OPN) plays a predominant regulatory role in expressing many well-known EMT activators, thus being recognized as a critical regulator of the entire process. Moreover, OPN can modify the tissue and tumor microenvironment to support EMT by generating cancer-associated fibroblasts $(82,83)$. The clinical significance of OPN as a biomarker for poor prognosis has been reported in GISTs; increased OPN expression was significantly associated with higher mitosis rate, poor recurrence prognosis, high-risk status and worse DFS (84). OPN, upon its interaction and upregulating effect on CD44 surface expression, was proved to contribute to tumor cell proliferation. CD44 is an OPN receptor highly expressed in the vast majority of malignancies and promotes processes involved in metastases via interaction with appropriate extracellular matrix ligands (85). High OPN expression and its interaction with CD44 is correlated with elevated mitosis rate in GIST tissues, possibly through subsequent downstream signaling contributing to the enhanced proliferation (84). Moreover, OPN elicits an anti-apoptotic effect through $\beta$-catenin-mediated upregulation of anti-apoptotic protein-induced myeloid leukemia cell differentiation protein (Mcl-1), and as a result, attenuates imatinib-induced apoptosis in GIST in vitro. The discussed mechanism might underly drug resistance to imatinib among some patients with GIST (14).

Another molecule affecting EMT and being studied in GIST is long non-coding RNA (lncRNA) AOC4P. AOC4P regulates EMT by affecting the production of vimentin, one of the EMT and metastasis markers. The expression of various EMT markers, including vimentin, transforming growth factor- $\beta 1$, ZEB1 and Snail, was significantly higher in GIST tissues compared with normal ones. In contrast, the expression of E-cadherin was found to be lower. This association was particularly significant in high-risk GIST cases. The downregulation of E-cadherin is the hallmark of the EMT in cancer. The decrease in E-cadherin leads to the exacerbation of GIST development, conversion of cytokeratin into vimentin, and consequently, EMT acceleration. The EMT process might be inhibited by AOC4P silencing that induces the increase in E-cadherin and the decrease in vimentin in carcinoma cells (86-88).

Slug, a member of the SNAIL family, is the most thoroughly investigated EMT regulator. Overexpression of Slug suppresses the expression of E-cadherin and increases the cancer cells' invasiveness (87). Approximately $90 \%$ of GIST cases might display SLUG overexpression. Nuclear positivity for SLUG is observed in GIST cases with distant metastasis, especially strong in extra-gastrointestinal ones (89). SLUG acts as a nuclear transcription factor and is more commonly expressed by large GISTs with pleomorphic nuclei and a high mitotic index. SLUG is described as an indicator of patients' unfavorable RFS. Downregulation of this member of the SNAIL family inhibits cell proliferation, induces cell death, and sensitizes GIST cells to the lower concentrations of imatinib (90). The molecules involved in the EMT are summarized in the Table $\mathrm{I}$.

\section{Conclusion}

Broadened knowledge considering GIST biology is a promising window for developing improved diagnostic and therapeutic strategies. Mutations in genes required for synapse and neural development may underlie some GIST behaviors, while miRNA dysregulation is an essential component in GIST expansion. On the other hand, resistance to imatinib may be associated with epithelial-mesenchymal transition. New molecules might be incorporated into risk stratification schemes due to their proven association with outcomes; however, further research is required. Therapies based on the significant role of angiogenesis, immunology and neural origin in the GIST biology could become a valuable enhancement of currently implemented treatment schemes. Although multiple obstacles must be defeated, developing an understanding of GIST carcinogenesis presents a promising future.

\section{Acknowledgements}

Not applicable.

\section{Funding}

Not applicable.

\section{Availability of data and materials}

Not applicable.

\section{Authors' contributions}

MMF and AMBK designed the study. MMF collected the data and researched the literature. MMF and AMBK drafted the manuscript. MMF and AMBK confirmed the authenticity of all the raw data. Both authors read and approved the final manuscript.

\section{Ethics approval and consent to participate}

Not applicable.

\section{Patient consent for publication}

Not applicable.

\section{Competing interests}

The authors declare that they have no competing interests.

\section{References}

1. Ahmed M: Recent advances in the management of gastrointestinal stromal tumor. World J Clin Cases 8: 3142-3155, 2020.

2. Yamamoto $\mathrm{H}$ and Oda $\mathrm{Y}$ : Gastrointestinal stromal tumor: Recent advances in pathology and genetics. Pathol Int 65: 9-18, 2015.

3. Hirota S: Differential diagnosis of gastrointestinal stromal tumor by histopathology and immunohistochemistry. Transl Gastroenterol Hepatol 3: 27, 2018.

4. Khoshnood A: Gastrointestinal stromal tumor-A review of clinical studies. J Oncol Pharm Pract 25: 1473-1485, 2019.

5. Kupcinskas J: Small molecules in rare tumors: Emerging role of MicroRNAs in GIST. Int J Mol Sci 19: 397, 2018.

6. Parab TM, DeRogatis MJ, Boaz AM, Grasso SA, Issack PS, Duarte DA, Urayeneza O, Vahdat S, Qiao JH and Hinika GS: Gastrointestinal stromal tumors: A comprehensive review. J Gastrointest Oncol 10: 144-154, 2019. 
7. Zhang $\mathrm{H}$ and Liu Q: Prognostic indicators for gastrointestinal stromal tumors: A review. Transl Oncol 13: 100812, 2020.

8. von Mehren M and Joensuu H: Gastrointestinal stromal tumors. J Clin Oncol 36: 136-143, 2018.

9. Shi YN, Li Y, Wang LP, Wang ZH, Liang XB, Liang H, Zhang L, Li B, Fan LQ, Zhao Q, et al: Gastrointestinal stromal tumor (GIST) with liver metastases: An 18-year experience from the GIST cooperation group in North China. Medicine (Baltimore) 96: e8240, 2017.

10. Li HL, Wang LH, Hu YL, Feng Y, Li XH, Liu YF, Li P, Mao QS and Xue WJ: Clinical and prognostic significance of CC chemokine receptor type 8 protein expression in gastrointestinal stromal tumors. World J Gastroenterol 26: 4656-4668, 2020.

11. Nishida T, Blay JY, Hirota S, Kitagawa Y and Kang YK: The standard diagnosis, treatment, and follow-up of gastrointestina stromal tumors based on guidelines. Gastric Cancer 19: 3-14, 2016

12. Schaefer IM, Marino-Enriquez A and Fletcher JA: What is new in gastrointestinal stromal tumor? Adv Anat Pathol 24: 259-267, 2017.

13. Den Hollander D, Van der Graaf WTA, Desar IME and Le Cesne A: Predictive factors for toxicity and survival of second-line sunitinib in advanced gastrointestinal stromal tumours (GIST). Acta Oncol 58: 1648-1654, 2019.

14. Hsu KH, Tsai HW, Lin PW, Hsu YS, Lu PJ and Shan YS Anti-apoptotic effects of osteopontin through the up-regulation of Mcl-1 in gastrointestinal stromal tumors. World J Surg Oncol 12: 189, 2014

15. Wei SC, Xu L, Li WH, Li Y, Guo SF, Sun XR and Li WW: Risk stratification in GIST: Shape quantification with CT is a predictive factor. Eur Radiol 30: 1856-1865, 2020.

16. Miettinen M and Lasota J: Gastrointestinal stromal tumors: Review on morphology, molecular pathology, prognosis, and differential diagnosis. Arch Pathol Lab Med 130: 1466-1478, 2006.

17. Di Vita M, Zanghi A, Cavallaro A, Cardì F, Uhlig M, Ursi P, Lo Menzo E, Panebianco V and Cappellani A: Gastric GIST and prognostic models. Which is the best to predict survival after surgery? Ann Ital Chir 90: 31-40, 2019.

18. Wong NA: Gastrointestinal stromal tumours--an update for histopathologists. Histopathology 59: 807-821, 2011.

19. Imamura M, Yamamoto H, Nakamura N, Oda Y, Yao T, Kakeji Y, Baba H, Maehara Y and Tsuneyoshi M: Prognostic significance of angiogenesis in gastrointestinal stromal tumor. Mod Pathol 20: 529-537, 2007.

20. Rey S, Schito L, Wouters BG, Eliasof S and Kerbel RS: Targeting Hypoxia-Inducible factors for antiangiogenic cancer therapy. Trends Cancer 3: 529-541, 2017.

21. Teleanu RI, Chircov C, Grumezescu AM and Teleanu DM: Tumor angiogenesis and anti-angiogenic strategies for cancer treatment. J Clin Med 9: 84, 2019

22. Liu N, Huang J, Sun S, Zhou Z, Zhang J, Gao F and Sun Q: Expression of matrix metalloproteinase-9, cyclooxygenase- 2 and vascular endothelial growth factor are increased in gastrointestinal stromal tumors. Int J Clin Exp Med 8: 6495-6501, 2015.

23. Tasdemir A, Soyuer I, Unal D and Artis T: Prognostic value of NF- $\kappa \mathrm{B}, \mathrm{CD}$, and VEGF in gastrointestinal stromal tumors. Contemp Oncol (Pozn) 17: 493-498, 2013

24. Basilio-de-Oliveira RP and Pannain VL: Prognostic angiogenic markers (endoglin, VEGF, CD31) and tumor cell proliferation (Ki67) for gastrointestinal stromal tumors. World J Gastroenterol 21: 6924-6930, 2015.

25. Kigure W, Fujii T, Sutoh T, Morita H, Katoh T, Yajima RN, Yamaguchi S, Tsutsumi S, Asao T and Kuwano H: The association of VEGF-C expression with tumor lymphatic vessel density and lymph node metastasis in patients with gastric cancer and gastrointestinal stromal tumor. Hepatogastroenterology 60: 277-280, 2013

26. Yang Z, Wang F, Liu S and Guan W: Comparative clinical features and short-term outcomes of gastric and small intestinal gastrointestinal stromal tumours: A retrospective study. Sci Rep 9: 10033, 2019.

27. Gromova P, Rubin BP, Thys A, Cullus P, Erneux C and Vanderwinden JM: ENDOGLIN/CD105 is expressed in KIT positive cells in the gut and in gastrointestinal stromal tumours. J Cell Mol Med 16: 306-317, 2012.

28. de Oliveira AT, Reis RM, Afonso J, Martinho O, Matos D, Carvalho AL, Vazquez VL, Silva TB, Scapulatempo C, Saad SS and Longatto-Filho A: Lymphangiogenic VEGF-C and VEGFR-3 expression in genetically characterised gastrointestinal stromal tumours. Histol Histopathol 26: 1499-1507, 2011
29. Hui Q, Jin Z, Li X, Liu C and Wang X: FGF family: From drug development to clinical application. Int J Mol Sci 19: 1875, 2018.

30. Boichuk S, Galembikova A, Mikheeva E, Bikinieva F, Aukhadieva A, Dunaev P, Khalikov D, Petrov S, Kurtasanov R, Valeeva E, et al: Inhibition of FGF2-Mediated Signaling in GIST-promising approach for overcoming resistance to imatinib. Cancers (Basel) 12: 1674, 2020

31. Sergei B, Pavel D, Aigul G, Firyuza B, Ilmira N, Ilshat M, Aida A, Refat K, Natalia A, Elena S and Vera G: Inhibition of FGFR2-Signaling Attenuates a Homology-Mediated DNA Repair in GIST and Sensitizes Them to DNA-Topoisomerase II Inhibitors. Int J Mol Sci 21: 352, 2020.

32. Li F, Huynh H, Li X, Ruddy DA, Wang Y, Ong R, Chow P, Qiu S, Tam A, Rakiec DP, et al: FGFR-Mediated Reactivation of MAPK signaling attenuates antitumor effects of imatinib in gastrointestinal stromal tumors. Cancer Discov 5: 438-451, 2015.

33. Li P, Cong Z, Qiang Y, Xiong L, Tang L, Zhang Y, Wu H, Yi J, Jing H, Li D and Shen Y: Clinical significance of CCBE1 expression in lung cancer. Mol Med Rep 17: 2107-2112, 2018

34. Tian GA, Zhu CC, Zhang XX, Zhu L, Yang XM, Jiang SH, Li RK, Tu L, Wang Y, Zhuang C, et al: CCBE1 promotes GIST development through enhancing angiogenesis and mediating resistance to imatinib. Sci Rep 6: 31071, 2016.

35. Mesci A, Huang X, Taeb S, Jahangiri S, Kim Y, Fokas E, Bruce J, Leong HS and Liu SK: Targeting of CCBE1 by miR-330-3p in human breast cancer promotes metastasis. Br J Cancer 116: 1350-1357, 2017.

36. Hirota S, Isozaki K, Moriyama Y, Hashimoto K, Nishida T, Ishiguro S, Kawano K, Hanada M, Kurata A, Takeda M, et al: Gain-of-function mutations of c-kit in human gastrointestinal stromal tumors. Science 279: 577-580, 1998

37. Wang CJ, Zhang ZZ, Xu J, Wang M, Zhao WY, Tu L, Zhuang C, Liu Q, Shen YY, Cao H and Zhang ZG: SLITRK3 expression correlation to gastrointestinal stromal tumor risk rating and prognosis. World J Gastroenterol 21: 8398-8407, 2015.

38. Bumming P, Nilsson O, Ahlman H, Welbencer A, Andersson MK, Sjölund K and Nilsson B: Gastrointestinal stromal tumors regularly express synaptic vesicle proteins: Evidence of a neuroendocrine phenotype. Endocr Relat Cancer 14: 853-863, 2007.

39. Ran L, Chen Y, Sher J, Wong EWP, Murphy D, Zhang JQ, Li D, Deniz K, Sirota I, Cao Z, et al: FOXF1 defines the core-regulatory circuitry in gastrointestinal stromal tumor. Cancer Discov 8: 234-251, 2018

40. Chi P, Chen Y, Zhang L, Guo X, Wongvipat J, Shamu T, Fletcher JA, Dewell S, Maki RG and Zheng D: ETV1 is a lineage survival factor that cooperates with KIT in gastrointestinal stromal tumours. Nature 467: 849-853, 2010.

41. Ran L, Sirota I, Cao Z, Murphy D, Chen Y, Shukla S, Xie Y, Kaufmann MC, Gao D, Zhu S, et al: Combined inhibition of MAP kinase and KIT signaling synergistically destabilizes ETV1 and suppresses GIST tumor growth. Cancer Discov 5: 304-315, 2015.

42. Hayashi Y, Bardsley MR, Toyomasu Y, Milosavljevic S, Gajdos GB, Choi KM, Reid-Lombardo KM, Kendrick ML, Bingener-Casey J, Tang CM, et al: Platelet-Derived Growth Factor Receptor- $\alpha$ Regulates proliferation of gastrointestinal stromal tumor cells with mutations in KIT by Stabilizing ETV1. Gastroenterology 149: 420-432.e16, 2015.

43. Sakamaki K, Funasaka K, Miyahara R, Furukawa K, Yamamura T, Ohno E, Nakamura M, Kawashima H, Hirooka Y, Fujishiro M and Goto H: Low ETV1 mRNA expression is associated with recurrence in gastrointestinal stromal tumors. Sci Rep 10: 14767, 2020.

44. Li J, Han W, Pelkey KA, Duan J, Mao X, Wang YX, Craig MT, Dong L, Petralia RS, McBain CJ and Lu W: Molecular Dissection of Neuroligin 2 and Slitrk3 reveals an essential framework for GABAergic Synapse Development. Neuron 96: 808-826.e8, 2017.

45. Agaimy A: Gastrointestinal stromal tumors (GIST) from risk stratification systems to the new TNM proposal: More questions than answers? A review emphasizing the need for a standardized GIST reporting. Int J Clin Exp Pathol 3: 461-471, 2010.

46. Hotzel J, Melling N, Muller J, Polonski A, Wolters-Eisfeld G, Izbicki JR, Karstens KF and Tachezy M: Protein expression of close homologue of L1 (CHL1) is a marker for overall survival in non-small cell lung cancer (NSCLC). J Cancer Res Clin Oncol 145: 2285-2292, 2019.

47. Karstens KF, Bellon E, Polonski A, Wolters-Eisfeld G, Melling N, Reeh M, Izbicki JR and Tachezy M: Expression and serum levels of the neural cell adhesion molecule L1-like protein (CHL1) in gastrointestinal stroma tumors (GIST) and its prognostic power. Oncotarget 11: 1131-1140, 2020. 
48. Thys A, Vandenberghe P, Hague P, Klein OD, Erneux C and Vanderwinden JM: Hyperplasia of interstitial cells of cajal in sprouty homolog 4 deficient mice. PLoS One 10: e0124861, 2015.

49. Vandenberghe P, Hague P, Hockman SC, Manganiello VC, DemetterP,Erneux Cand Vanderwinden JM: Phosphodiesterase 3A: A new player in development of interstitial cells of Cajal and a prospective target in gastrointestinal stromal tumors (GIST). Oncotarget 8: 41026-41043, 2017.

50. Lu TX and Rothenberg ME: MicroRNA. J Allergy Clin Immunol 141: 1202-1207, 2018.

51. Dragomir M, Mafra ACP, Dias SMG, Vasilescu C and Calin GA: Using microRNA networks to understand cancer. Int J Mol Sci 19: 1871, 2018

52. Isosaka M, Niinuma T, Nojima M, Kai M, Yamamoto E, Maruyama R, Nobuoka T, Nishida T, Kanda T, Taguchi T, et al: A screen for epigenetically silenced microRNA genes in gastrointestinal stromal tumors. PLoS One 10: e0133754, 2015.

53. Wang Y, Li J, Kuang D, Wang X, Zhu Y, Xu S, Chen Y, Cheng H, Zhao Q, Duan Y and Wang G: MiR-148b-3p functions as a tumor suppressor in GISTs by directly targeting KIT. Cell Commun Signal 16: 16, 2018.

54. Fan R, Zhong J, Zheng S, Wang Z, Xu Y, Li S, Zhou J and Yuan F: MicroRNA-218 inhibits gastrointestinal stromal tumor cell and invasion by targeting KIT. Tumour Biol 35: 4209-4217, 2014.

55. Yun S, Kim WK, Kwon Y, Jang M, Bauer S and Kim H: Survivin is a novel transcription regulator of KIT and is downregulated by miRNA-494 in gastrointestinal stromal tumors. Int J Cancer 142: 2080-2093, 2018.

56. Fan R, Zhong J, Zheng S, Wang Z, Xu Y, Li S, Zhou J and Yuan F: MicroRNA-218 increase the sensitivity of gastrointestinal stromal tumor to imatinib through PI3K/AKT pathway. Clin Exp Med 15: 137-144, 2015

57. Niinuma T, Suzuki H, Nojima M, Nosho K, Yamamoto H, Takamaru H, Yamamoto E, Maruyama R, Nobuoka T, Miyazaki Y, et al: Upregulation of miR-196a and HOTAIR drive malignant character in gastrointestinal stromal tumors. Cancer Res 72: 1126-1136, 2012.

58. Niinuma $\mathrm{T}$, Kai M, Kitajima $H$, Yamamoto E, Harada $\mathrm{T}$, Maruyama R, Nobuoka T, Nishida T, Kanda T, Hasegawa T, et al: Downregulation of miR-186 is associated with metastatic recurrence of gastrointestinal stromal tumors. Oncol Lett 14: 5703-5710, 2017.

59. Anastasiadou E, Jacob LS and Slack FJ: Non-coding RNA networks in cancer. Nat Rev Cancer 18: 5-18, 2018.

60. Awasthi R, Rathbone MJ, Hansbro PM, Bebawy M and Dua K Therapeutic prospects of microRNAs in cancer treatment through nanotechnology. Drug Deliv Transl Res 8: 97-110, 2018.

61. Mansoori B, Mohammadi A, Shirjang S and Baradaran B MicroRNAs in the diagnosis and treatment of cancer. Immunol Invest 46: 880-897, 2017.

62. Catela Ivkovic T, Voss G, Cornella H and Ceder Y: MicroRNAs as cancer therapeutics: A step closer to clinical application. Cancer Lett 407: 113-122, 2017.

63. Rusakiewicz S, Semeraro M, Sarabi M, Desbois M, Locher C, Mendez R, Vimond N, Concha A, Garrido F, Isambert N, et al: Immune infiltrates are prognostic factors in localized gastrointestinal stromal tumors. Cancer Res 73: 3499-3510, 2013.

64. Tan Y, Garcia-Buitrago MT, Trent JC and Rosenberg AE: The immune system and gastrointestinal stromal tumor: A wealth of opportunities. Curr Opin Oncol 27: 338-342, 2015.

65. Tan Y, Trent JC, Wilky BA, Kerr DA and Rosenberg AE: Current status of immunotherapy for gastrointestinal stromal tumor. Cancer Gene Ther 24: 130-133, 2017.

66. Balachandran VP, Cavnar MJ, Zeng S, Bamboat ZM, Ocuin LM, Obaid H, Sorenson EC, Popow R, Ariyan C, Rossi F, et al: Imatinib potentiates antitumor $T$ cell responses in gastrointestinal stromal tumor through the inhibition of Ido. Nat Med 17: 1094-1100, 2011.

67. Seifert AM, Zeng S, Zhang JQ, Kim TS, Cohen NA, Beckman MJ, Medina BD, Maltbaek JH, Loo JK, Crawley MH, et al: PD-1/PD-L1 Blockade Enhances T-cell activity and antitumor efficacy of imatinib in gastrointestinal stromal tumors. Clin Cancer Res 23: 454-465, 2017

68. Bertucci F, Finetti P, Mamessier E, Pantaleo MA, Astolfi A, Ostrowski J and Birnbaum D: PDL1 expression is an independent prognostic factor in localized GIST. Oncoimmunology 4: e1002729, 2015.

69. Zhao R, Song Y, Wang Y, Huang Y, Li Z, Cui Y, Yi M, Xia L, Zhuang W, Wu X and Zhou Y: PD-1/PD-L1 blockade rescue exhausted CD8+ T cells in gastrointestinal stromal tumours via the PI3K/Akt/mTOR signalling pathway. Cell Prolif 52: e12571, 2019.
70. Toulmonde M,Penel N, Adam J, Chevreau C, Blay JY,Le Cesne A, Bompas E, Piperno-Neumann S, Cousin S, Grellety T, et al: Use of PD-1 Targeting, Macrophage Infiltration, and IDO pathway activation in sarcomas: A Phase 2 clinical trial. JAMA Oncol 4: 93-97, 2018

71. Wang HC, Li TY, Chao YJ, Hou YC, Hsueh YS, Hsu KH and Shan YS: KIT Exon 11 Codons 557-558 deletion mutation promotes liver metastasis through the CXCL12/CXCR4 axis in gastrointestinal stromal tumors. Clin Cancer Res 22: 3477-3487, 2016.

72. De Simone M, Arrigoni A, Rossetti G, Gruarin P, Ranzani V, Politano C, Bonnal RJP, Provasi E, Sarnicola ML, Panzeri I, et al: Transcriptional landscape of human tissue lymphocytes unveils uniqueness of Tumor-Infiltrating T regulatory cells. Immunity 45: 1135-1147, 2016.

73. Tanaka A and Sakaguchi S: Regulatory T cells in cancer immunotherapy. Cell Res 27: 109-118, 2017.

74. Soler D, Chapman TR, Poisson LR, Wang L, Cote-Sierra J, Ryan M, McDonald A, Badola S, Fedyk E, Coyle AJ, et al: CCR8 expression identifies CD4 memory T cells enriched for FOXP3+ regulatory and Th2 effector lymphocytes. J Immunol 177: 6940-6951, 2006.

75. Sun X, Cheng G, Hao M, Zheng J, Zhou X,Zhang J, Taichman RS, Pienta KJ and Wang J: CXCL12 / CXCR4 / CXCR7 chemokine axis and cancer progression. Cancer Metastasis Rev 29: 709-722, 2010.

76. Yesilkanal AE and Rosner MR: Targeting Raf kinase inhibitory protein regulation and function. Cancers (Basel) 10: 306, 2018.

77. Wang Y, Chen JJ, Wang XF and Wang Q: Clinical and prognostic significance of Raf kinase inhibitory protein expression in gastrointestinal stromal tumors. World J Gastroenterol 24: 2508-2517, 2018

78. Yesilkanal AE and Rosner MR: Raf kinase inhibitory protein (RKIP) as a metastasis suppressor: Regulation of signaling networks in cancer. Crit Rev Oncog 19: 447-454, 2014.

79. Valadao M, Braggio D, Santos AF, Pimenta-Inada HK, Linhares E, Gonçalves R, Romano S, Vilhena B, Small I, Cubero D, et al: Involvement of signaling molecules in the prediction of response to imatinib treatment in metastatic GIST patients. J Surg Res 178: 288-293, 2012.

80. Zhang Y and Weinberg RA: Epithelial-to-mesenchymal transition in cancer: Complexity and opportunities. Front Med 12: 361-373, 2018.

81. Dongre A and Weinberg RA: New insights into the mechanisms of epithelial-mesenchymal transition and implications for cancer. Nat Rev Mol Cell Biol 20: 69-84, 2019.

82. Kothari AN, Arffa ML, Chang V, Blackwell RH, Syn WK, Zhang J, Mi Z and Kuo PC: Osteopontin-A master regulator of Epithelial-Mesenchymal transition. J Clin Med 5: 39, 2016.

83. Shevde LA and Samant RS: Role of osteopontin in the pathophysiology of cancer. Matrix Biol 37: 131-141, 2014.

84. Hsu KH, Tsai HW, Lin PW, Hsu YS, Shan YS and Lu PJ: Osteopontin expression is an independent adverse prognostic factor in resectable gastrointestinal stromal tumor and its interaction with CD44 promotes tumor proliferation. Ann Surg Oncol 17: 3043-3052, 2010

85. Senbanjo LT and Chellaiah MA: CD44: A multifunctional cell surface adhesion receptor is a regulator of progression and metastasis of cancer cells. Front Cell Dev Biol 5: 18, 2017.

86. Hu JC, Wang Q, Jiang LX, Cai L, Zhai HY, Yao ZW, Zhang ML and Feng Y: Effect of long non-coding RNA AOC4P on gastrointestinal stromal tumor cells. Onco Targets Ther 11: 6259-6269, 2018.

87. Zhang K, Lu C, Huang X, Cui J, Li J, Gao Y, Liang W, Liu Y, Sun Y, Liu $\mathrm{H}$, et al: Long noncoding RNA AOC4P regulates tumor cell proliferation and invasion by epithelial-mesenchymal transition in gastric cancer. Therap Adv Gastroenterol 12: 1756284819827697, 2019.

88. Hao Y, Baker D and Ten Dijke P: TGF-beta-Mediated Epithelial-Mesenchymal transition and cancer metastasis. Int J Mol Sci 20: 2767, 2019.

89. Kovecsi A, Gurzu S, Szentirmay Z, Kovacs Z, Bara TJ and Jung I: Paradoxical expression pattern of the epithelial mesenchymal transition-related biomarkers CD44, SLUG, N-cadherin and VSIG1/Glycoprotein A34 in gastrointestinal stromal tumors. World J Gastrointest Oncol 9: 436-443, 2017.

90. Pulkka OP, Nilsson B, Sarlomo-Rikala M, Reichardt P, Eriksson M, Hall KS, Wardelmann E, Vehtari A, Joensuu H and Sihto H: SLUG transcription factor: A pro-survival and prognostic factor in gastrointestinal stromal tumour. Br J Cancer 116: $1195-1202,2017$. 